\title{
Analytical and empirical modelling of the origin and heliospheric propagation of coronal mass ejections, and space weather applications
}

\author{
Bojan Vršnak* \\ Hvar Observatory, Faculty of Geodesy, University of Zagreb, Kačićeva 26, HR-10000 Zagreb, Croatia
}

Received 27 October 2020 / Accepted 19 March 2021

\begin{abstract}
The focus is on the physical background and comprehension of the origin and the heliospheric propagation of interplanetary coronal mass ejections (ICMEs), which can cause most severe geomagnetic disturbances. The paper considers mainly the analytical modelling, providing useful insight into the nature of ICMEs, complementary to that provided by numerical MHD models. It is concentrated on physical processes related to the origin of CMEs at the Sun, their heliospheric propagation, up to the effects causing geomagnetic perturbations. Finally, several analytical and statistical forecasting tools for space weather applications are described.
\end{abstract}

Keywords: coronal mass ejections / heliospheric propagation / geomagnetic storms / forecasting tools

\section{Introduction}

Solar sub-photospheric convection, differential rotation, and surface flows, in the interaction with solar magnetic field act as a magnetohydrodynamic (MHD) dynamo, inducing electric currents and thus transforming the mechanical energy into the electromagnetic one. The free magnetic energy associated with the induced currents is partly spent for (gradual/instantaneous) coronal heating, and partly is accumulating in large-scale coronal magnetic structures that can generally be described as coronal arcades, magnetic loops, or flux ropes.

Coronal heating is a result of small-scale energy releases either in the form of nano-flares, alternating currents (MHD waves), or persistent Joule heating by direct currents (for a review see, e.g., Parnell \& De Moortel, 2012). The required energy comes from the subphotospheric convective mechanical work that stresses the magnetic field, which transports the electromagnetic energy into the corona, enabling the heating of the corona above the much cooler solar surface. The process is more efficient in regions of stronger magnetic field, which is clearly seen from the fact that sunspots, which are colder than the surrounding photosphere, are related to coronal structures that are hotter than the ambient corona. It should also be noted that the process of coronal heating is tightly related to the generation of solar wind. In this respect, it should be noted that changes in the solar wind conditions can significantly influence the space weather. For example, high-speed streams (HSSs) and the associated corotating interaction regions (CIRs), originating from equatorial coronal holes, can significantly affect the state of the magnetosphere, causing weak or moderate geomagnetic storms (e.g. Tsurutani et al., 1995, 2006a, b; Verbanac et al., 2011a, and references therein) and modulating the flux of incoming cosmic rays (e.g., Dumbović et al., 2012; Vršnak, 2016, and references therein). The cumulative effects of HSSs/CIRs, in fact, can be the most significant effect on the near-Earth space weather (Tsurutani et al., 2006b).

The energy that is accumulated in the large-scale coronal magnetic structures cannot increase indefinitely. Thus, when the system becomes overloaded, it loses its equilibrium and the accumulated energy is explosively released in the form of coronal mass ejections (CMEs), solar flares, various forms of coronal jets, etc. These powerful processes strongly affect the space weather, as they result in solar energetic particles, soft- and hard-X rays, increased EUV radiation, MHD shocks, and interplanetary CMEs (ICMEs) that transport strong fields into the heliosphere, etc. (for a review see, e.g., Schwenn, 2006). All these phenomena strongly affect the Earth magnetic field, ionosphere, and even the thermosphere (e.g., Knipp et al., 2004; Pulkkinen, 2007;

*Corresponding author: bvrsnak@geof.hr 
Krauss et al., 2015; Koskinen et al., 2017; Tsurutani et al., 2020). ICMEs, i.e., the interplanetary successors of CMEs, cause the most severe transient disturbances in the heliosphere and at the Earth, where the ICME-related effects can strongly influence our everyday life (e.g., Feynman \& Gabriel, 2000; Schrijver et al., 2015). Consequently, the understanding of the physical nature of their origin, dynamics, and heliospheric propagation is one of the central points of the space weather science, and space weather forecasting. In this paper we first focus on the physical background and the nature of CMEs (Sect. 2). Then, their heliospheric propagation is discussed (Sect. 3), and towards the end of the paper, some examples of the empirical and analytical models that are frequently used in forecasting the CME arrival time, impact speed, and geoeffectiveness are presented (Sect. 4). Finally, in Section 5 some concluding remarks are given. What is written below is a kind of "personal review" of our comprehension of the CME/ICME physics, particularly bearing in mind the population of young scientists, i.e., the paper is written in a such a way to help them in the early stages of their scientific careers.

\section{Physics of coronal mass ejections}

Although the pre-eruptive coronal features as well as the eruption itself generally can have a very complex structure, the eruption of CMEs is nowadays usually attributed to a destabilization of a coronal magnetic arcade embedding a semi-toroidal magnetic flux rope, anchored at both sides in the opposite photospheric magnetic polarities (e.g., Chen, 1989; Vršnak, 1990; Titov \& Démoulin, 1999; Török \& Kliem, 2005); for a review see, e.g., Schmieder et al. (2015); Green et al. (2018). This simple concept is based on the fact that the coronal structure must contain free magnetic energy, i.e., it has to contain electric currents, in order to be accelerated into the upper corona and interplanetary space. In the pre-eruptive phase, when the structure is still stable, the currents are co-aligned with the magnetic field, i.e., the structure is in the force-free state. Such a concept is supported by the observations, where pre-eruptive, as well as eruptive features usually show the so-called three-part structure: the bright frontal rim, the cavity and the bright core. These features are usually interpreted as signatures of the the overlying arcade, low-density flux-rope seen as its cross-section, and a dense prominence material at the bottom of the cavity, respectively (e.g., Gibson et al., 2006 and references therein). For analogous pre-eruptive signatures see, e.g., Engvold (1988). Finally, regarding the pre-eruptive magnetic field structure, it should be emphasized that a flux rope is not a necessary ingredient for the eruption; many authors consider also a sheared arcade or various quadrupolar structures (for observational aspects see, e.g., Song et al., 2014; Schmieder et al., 2015; Ouyang et al., 2017; Nindos et al., 2020, for theoretical aspect see, e.g., Hood \& Anzer 1987 and references therein), where the flux rope is formed in the course of eruption. In the following we focus on the pre-eruptive flux rope embedded in a coronal arcade.

\subsection{Initiation and the main acceleration phase}

Mostly, the eruption itself is preceded by swelling and slow rising motion of the pre-eruptive structure. We know for at least two different processes that cause such a pre-eruptive behaviour. The first one is gradual evolution of the pre-existing coronal structure through a series of quasi-equilibrium states, most clearly seen in the case of eruptive prominences. This process is related to the Poynting flux $E \times B$ that brings free magnetic energy into the corona due to, e.g., twisting and braiding motions of flux-rope footpoints, shearing motions of the arcade footpoints, so-called flux cancellation at the overlying arcade footpoints caused by converging motions of opposite magnetic polarities, or directly to the emergence of new magnetic flux. All these processes result in the increase of the flux-rope electric current, and can lead to the loss of equilibrium of the whole structure, and consequently, the eruption (e.g., Vršnak, 2008, 2016, 2019)

The second process is related to the emergence of an already unstable magnetic flux rope from the convective zone, trespassing the photosphere, chromospheric layers and the transition region towards the corona. In this case there are two reasons why the gradual pre-eruption phase occurs. First, the flux-rope emergence itself is slow due to large inertia of the dense plasma contained within the flux rope, so it cannot be rapidly accelerated and thus, it does not move fast. Second, the eruption can be initiated only after most of closed magnetic field in the flux rope emerges into the low-density corona. During this emergence, all the way through the chromosphere up to corona, the dense flux-rope plasma leaks from the flux rope downwards due to gravity, and the structure gradually becomes lighter. At a given moment, when the conditions for the eruptions are fulfilled, the structure starts to rapidly accelerate and erupts.

Generally, in both described processes, the eruption-associated flare energy release occurs with a little delay after the beginning of the eruption, when already a measurable acceleration occurs (e.g., Berkebile-Stoiser et al., 2012). However, there are cases when the energy release in the form of the so-called confined solar flare is observed prior to the eruption acceleration phase (e.g., Aurass et al., 1999; Nindos et al., 2020). In these cases, the evolution of the pre-eruptive structure is basically not gradual, rather the transition to the unstable state goes quite rapidly due to the flare-related fast restructuring of the preeruptive magnetic field configuration.

Finally, it should be noted that the mentioned processes are just the most common scenarios that can be inferred from observations. Yet, there are also other possible processes in complex coronal structures, such as, e.g., interactions of neighbouring large-scale coronal structures, interaction with coronal hole boundaries, destabilization of meta-stable structures by MHD shock waves coming from distant eruptions, etc.

After the pre-eruptive structure loses its equilibrium, it starts to accelerate under the action of unbalanced Lorentz force. It can be caused either directly by the so-called hoop force (e.g., Chen, 1989; Vršnak, 1990, and references therein), or can be due to some of well-known instabilities such as kink or torus instability, or can be due to a combination of all of them (for a review see, e.g., Schmieder et al., 2015; Green et al., 2018).

\subsection{Role of reconnection in CME-associated flares}

As shown by Vršnak (2008), the ideal-MHD processes (processes not including the resistivity-related effects), generally can result in accelerations up to several hundreds of $\mathrm{m} \mathrm{s}^{-2}$, i.e., cannot come to the $\mathrm{km} \mathrm{s}^{-2}$ range, even in most extreme preeruption magnetic field configurations (e.g., extremely large 
twists, favourable overlying-field conditions, etc.; for details see Sect. 2.2.3 in Green et al., 2018, and references therein). The main reason lies in the fact that the self-inductivity $L$ of any expanding structure increases, as $L$ is generally proportional to the dimensions of the system (Garren \& Chen, 1994; Žic et al., 2007). On the other hand, the magnetic flux associated with the electric current $I$ flowing through the system, $\Phi=L I$, in the absence of resistive processes, e.g., magnetic reconnection, remains preserved. Thus, if $L$ increases and $\Phi=$ const., the current $I$ has to decrease. Consequently, the Lorentz force gradually ceases, and the acceleration weakens. Indeed, observations indicate that, generally, the Lorentz force decreases as the heliocentric distance of CMEs increases (Vršnak, 2001).

However, the mentioned principles concern only the idealMHD processes, and the situation changes if resistive processes break the $\Phi=$ const. condition. In this respect, it should be noted that there is a simple, but very important, observational fact evidencing that resistive processes, better to say reconnection, are tightly related to the acceleration phase of CMEs. That is, CMEs are often associated with solar flares, which occur in low corona below the erupting CME, and are caused by magnetic reconnection (Miklenic et al., 2007, 2009) in the current sheet that is caused by the CME rising motion (Ko et al., 2003; Bemporad et al., 2006; Veronig et al., 2006; Vršnak et al., 2009). Statistically, faster CMEs are associated with more powerful flares (Bein et al., 2012; Veronig et al., 2018) and more impulsively accelerated CMEs are associated with more impulsive flares (Bein et al., 2012). It has also been shown that CMEs with higher accelerations are associated with hard-Xray flares that are characterized by harder electron spectra (Berkebile-Stoiser et al., 2012). Moreover, the flare-related energy release is closely synchronized with the CME acceleration (e.g., Vršnak et al., 2004a; Maričić et al., 2007; Temmer et al., 2008, 2010; Bein et al., 2012; Berkebile-Stoiser et al., 2012; Veronig et al., 2018). The most direct evidence of the relationship between the CME acceleration and the reconnection was presented by Miklenic et al. (2009), who compared the inferred reconnection rate and the $\mathrm{CME}$ kinematics and found a clear correlation between the total reconnected flux and the CME velocity (see also the statistical studies by Qiu \& Yurchyshyn, 2005; Tschernitz et al., 2018).

The explanation for this CME-flare coupling lies in the fact that the reconnection below the rising CME supplies the expanding flux rope with additional poloidal flux, which has two important effects. First, it reinforces the outward directed component of the hoop force, thus providing a stronger net Lorentz force and consequently, a stronger acceleration. Second, it reduces the inductive effects that tend to decrease the electric current in the flux rope due to the increasing size of the CME, i.e., it supports the current and thus prolongs the action of the Lorentz force and enables longer acceleration phase (for details see Vršnak, 2008, 2009, 2016).

Yet, the story does not end here. As a matter of fact, there is a feed-back relationship between the CME acceleration and the flare-related reconnection in the wake of CME. Namely, a higher CME acceleration creates stronger flows of plasma from regions ahead of the flux rope to its rear, which causes vortex-type of motion, pushing the plasma into the rarefied regions behind the flux rope, thus reinforcing reconnection (Vršnak, 2008, 2016; Temmer et al., 2010). This explains a tight synchronization of the CME acceleration and the flare-related energy release.
Statistically, fast and impulsively-accelerated CMEs originate from strong-field regions, and start to accelerate at low heights (Vršnak, 2001; Vršnak et al., 2007; Bein et al., 2011). This is consistent with the hypothesis that stronger CME accelerations are driven by stronger magnetic fields, as the Lorentz force is the main driver of the eruption. Thus, one can say that the kinetic energy of the eruption comes from the free energy stored in the magnetic field, and as the free-energy is just a part of total energy (another part, related to the potential field cannot be converted to work), it can be concluded that $\rho v^{2} / 2<B^{2} / 2 \mu_{0}$, i.e., that the CME kinetic energy density cannot exceed the total magnetic energy density, implying $v_{\mathrm{cme}}<v_{\mathrm{A}}$, where $v_{\mathrm{A}}$ represents the Alfvén speed within the CME body (for details see Vršnak, 2008, and Sect. 2.2.3 in Green et al., 2018). Thus, in stronger fields an eruptive structure can basically achieve a higher speed.

Yet, it should be noted that sometimes, in the case of eruptions of huge quiescent prominences, although the eruption comes from quiet regions and somewhat larger heights, can also achieve high speeds, exceeding $1000 \mathrm{~km} \mathrm{~s}^{-1}$ (Vršnak et al., $2005 b)$. The reason lies in the fact that the pre-eruption Lorentz force must be quite strong to balance the action of gravity on the dense prominence plasma (note that here the structure is in a strongly non-force-free state). When such structure loses equilibrium and starts to accelerate, the acceleration is relatively weak due to large inertia of the prominence. However, as the prominence rises and the magnetic field stretches, causing flattening of "dips" in the field structure where the prominence plasma is "floating", the prominence material starts to drain downward along legs of the prominence-carrying flux rope due to the gravity. The eruptive structure becomes lighter an lighter, providing continuously enhancing acceleration and eventually, leads to quite high speeds. Of course, as the described process takes quite some time, the acceleration is rather gradual, and the eruption achieves a high velocity quite late, at quite large heights.

\subsection{Kinematics in the outer corona}

Although the described processes and their relationships tend to prolong the action of the Lorentz force, it nevertheless weakens with the increasing time/distance (Vršnak, 2001). If nothing else, this happens because the magnetic field weakens with the heliospheric distance. On the other hand, the MHDdrag effects (Cargill et al., 1994, 1996; Cargill, 2004) increase, as the speed of fast CMEs increases and such CMEs may become faster than the ambient solar wind.

The MHD drag is a consequence of the transfer of the momentum and kinetic energy to the ambient upstream solarwind magnetoplasma that is permanently pushed outwards by faster-than-wind CMEs. The acceleration caused by the MHD drag can be approximately expressed in a form analogous to that describing the aerodynamic drag (Cargill, 2004):

$$
a=-\gamma(v-w)|v-w|
$$

Here, $v$ is the CME speed and $w$ the ambient solar wind speed. The "drag parameter" $\gamma$ (Vršnak et al., 2013, see also Vršnak et al., 2008) is usually expressed as $\gamma=c_{\mathrm{d}} A \rho_{\mathrm{w}} / M_{\text {tot }}$, where $A$ is the CME cross-section area, and $\rho_{\mathrm{w}}$ is the ambient plasma density. The total mass, $M_{\mathrm{tot}}$, is the sum of the mass of ejection, 
$M_{\text {cme }}$, and the so-called virtual mass, $M_{\mathrm{v}}$, that is related to the mass piled-up by the ejection. The virtual mass can be approximately estimated as $M_{\mathrm{v}}=\rho_{\mathrm{w}} V / 2$, where $V$ is the volume of the ICME (for details see Cargill, 2004, and references therein). Thus, the total mass $M_{\mathrm{tot}}=M_{\mathrm{cme}}+M_{\mathrm{v}}$ represents the mass of the ejection and the mass pilled up by the interaction of the ejection with the ambient plasma (Fig. 1a). The MHD nature of equation (1), which is basically analogous to the air-drag, is hidden in the dimensionless drag coefficient $c_{\mathrm{d}}$ (Cargill, 2004). It can vary from $c_{\mathrm{d}} \ll 1$ when reconnection is involved, to $c_{\mathrm{d}} \approx 10$ when the density of the ejection is lower than the ambient density, and also depends on the orientation of the magnetic field relative to the ejection (for details see, Cargill, 2004). In the case of slower-than-wind CMEs, the mass piles up at the rear of the CME (Fig. 1b) and the momentum is delivered by the wind to the CME. Equation (1) states that the drag tends to decelerate fast CMEs, as $v>w$ means $a<0$, and should accelerate slow CMEs, i.e., when $v<w$, then $a>0$.

Consequently, at a certain speed of fast CMEs, at a certain height range, the Lorentz force and the drag force become equal. In this phase, when the two forces are approximately equally strong, and thus there is practically no acceleration to be observed, the CME moves at an approximately constant speed.

Thus, after the main acceleration phase the eruption enters into the regime of "residual acceleration", as named by Zhang $\&$ Dere (2006). Observations in the SOHO/LASCO C2 and $\mathrm{C} 3$ field-of-view $(\approx 2-30$ solar radii) show that the $\mathrm{CME}$ accelerations are centered at 0 , implying a largely constant velocity in the outer corona (Vršnak et al., 2004b; Zhang \& Dere, 2006). Still a detailed inspection of the data reveal that in this stage, fast CMEs mostly decelerate, whereas those that did not achieve the solar wind speed during the main acceleration stage, usually show a weak gradual acceleration. In this stage the Lorentz force becomes negligible, so basically only the drag governs the CME motion (Vršnak, 2001). However, it should be noted that even some fast CMEs are still significantly accelerating within the coronagraphic field of view (Vršnak et al., 2004b), indicating a prolonged action of the Lorentz force. It is also worth mentioning that practically no slow CMEs that decelerate at low speeds have been recorded (Vršnak et al., 2004b).

As already mentioned, if a CME is faster than the ambient solar wind, beyond a certain height range that can be sometimes up to $\approx 100$ solar radii (Temmer et al., 2011; Manchester et al., 2017), the drag acceleration becomes a dominant force. Let us also note that the drag parameter $\gamma$ should be approximately constant over a large distance range, since in the interplanetary space $\rho \propto 1 / r^{2}$ and $A \propto r^{2}$, where $r$ is the heliocentric distance. However, due to deceleration, the CME speed decreases and becomes closer and closer to the solar-wind speed so the net deceleration weakens and after a while the CME moves at approximately constant speed (e.g., Gopalswamy et al., 2001; Reiner et al., 2007).

In the case of CMEs that are slower than the ambient solar wind, equation (1) shows that the drag acts so that it accelerates them until they achieve a speed comparable with the solar-wind speed. In this case, the solar wind pushes the CME from behind, creating a pile-up of solar wind plasma at the rear of the CME (Fig. 1b). Unlike in the case of fast CMEs, where the CME delivers the momentum to the ambient plasma, in this case the solar wind transfers its momentum to the CME, increasing its kinetic energy. a)
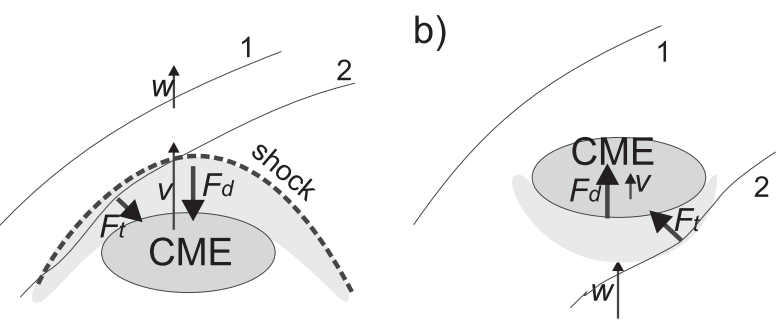

Fig. 1. Global effects acting on the ICMEs dynamics after the driving Lorentz force becomes negligible. The main component of the drag force is denoted as $F_{\mathrm{d}}$, whereas $F_{\mathrm{t}}$ depicts the force caused by the tension and magnetic pressure gradient resulting from the ambient field deformation. a) ICME faster than the ambient solar wind $(v>w)$ with a pile up of mass ahead (light gray). b) ICME slower than the ambient solar wind $(v<w)$ with a pile up of mass behind. East is to the right, west to the left.

\section{Heliospheric propagation}

The presented considerations are consistent with findings summarized by Gopalswamy et al. (2001); Gopalswamy (2006), demonstrating that the heliospheric propagation of CMEs (at large heliospheric distances we say interplanetary CMEs or simply, ICMEs) is characterized by deceleration of fast ICMEs and acceleration of slow ones, indirectly confirming a dominance of the drag in the ICME propagation. The importance of the drag, already during the coronal propagation of CMEs, was statistically demonstrated by Vršnak et al. (2004b). It was shown therein that in vast majority of events, fast CMEs decelerate and slow accelerate already below $\approx 30$ solar radii, and moreover, that the fit in the accelerationversus-speed graph, which included more than 5000 events, is better if the form given by equation (1) is applied, than, e.g., if the linear fit is used. For a recent review concerning the heliospheric propagation of ICMEs see, e.g., Manchester et al. (2017).

\subsection{Drag}

Equation (1) describes an approximate behaviour of the MHD drag, which is in its form analogous to the widely used expression for the standard aerodynamic drag. The difference is in the dimensionless constant $c_{\mathrm{d}}$ that comes from the MHD approach (for details see, Cargill, 2004). As already mentioned, if $v>w$ the moving structure decelerates, as the acceleration is $a<0$. If $v<w$, it accelerates, as the acceleration is $a>0$. If $v=w$, it moves at a constant speed, as there is no acceleration, $a=0$.

The drag parameter $\gamma$ says that the drag is more effective in the events that are wider, i.e., $A$ is large. Also, it is larger in denser ambience. The presented form for $\gamma$ can be transformed, showing that $\gamma$ is approximately proportional to the ratio of the ambient solar wind density and the CME density, $\rho_{\mathrm{w}} / \rho_{\mathrm{cme}}$ (for details see, Cargill, 2004; Vršnak et al., 2013).

The value of $\gamma$ can be estimated from observations by fitting equation (1) to the observed ICME kinematics, if the value of the actual solar wind speed is at least approximately known. Anyway, in fitting, the value of $w$ can be varied over a certain 
reasonable range of values, providing the range of possible values of $\gamma$. Most often, $\gamma$ is the range from $0.1 \times 10^{-7} \mathrm{~km}^{-1}$ (generally in the solar minimum) up to $2.5 \times 10^{-7} \mathrm{~km}^{-1}$ (in the solar maximum and in complex solar wind configurations), with the mean value of $\approx 1 \times 10^{-7} \mathrm{~km}^{-1}$ (for a distribution of values see Vršnak et al., 2013). If nothing else, this is because CMEs can have quite different masses, ranging from $\approx 10^{12} \mathrm{~g}$ to $>10^{16} \mathrm{~g}$, with a peak in the distribution around $\approx 4 \times 10^{14} \mathrm{~g}$ (e.g., Gopalswamy et al., 2009).

The drag coefficient $c_{\mathrm{d}}$ is generally of a not-well-known value. It depends on the relative orientation of the ambient solar-wind magnetic field as well as on the level of the MHD turbulence, and most of all, on the density ratio $\rho_{\mathrm{w}} / \rho_{\mathrm{cme}}$ (for a discussion and comparison with the observations, see, Cargill et al., 1996; Cargill, 2004). The value of $c_{\mathrm{d}}$ increases from $\approx 1$ for dense ICMEs to $>1$ for tenuous ones. Note that the value of $c_{\mathrm{d}}$ in the solar wind basically can be inferred from observations, if they allow an estimate of $A, \rho_{\mathrm{w}}, M_{\mathrm{tot}}$, and $w$, and of course, reliable measurements of the ICME kinematics. However, this was not done yet.

Finally, it is important to note that if we assume that the solar wind is approximately isotropic (which is, by the way, a quite questionable approximation) the value of $\gamma$ is constant during the heliospheric propagation, since $\rho_{\mathrm{w}} \propto r^{-2}$ due to $w \approx$ const., and $A \propto r^{2}$.

\subsection{Additional relevant effects}

There are various phenomena that in addition to the basic, most general behaviour described by equation (1), affect the ICME motion. These are various forms of deflections, CMECME interactions, and CME interactions with the solar-wind high-speed streams originating from coronal holes, which can significantly alter the CME heliospheric kinematics and propagation direction.

Regarding the ICME deflections one should primarily note that there is one effect that influences fast as well as slow ICMEs over large range of distances. Namely, the interplanetary field has the shape of Archimed spiral (in the solar wind case, often called Parker spiral) due to the interplay of radially expanding solar wind and the rotation of the solar surface, where the field lines are rooted. When a fast CME that initially expands radially, propagates through the inclined magnetic field of a Parker spiral, it deforms the field lines, causing the magnetic tension and pressure gradient that both act in the eastward direction, and thus the ICME is deflected to the east (so-called "eastward deflection", Wang et al., 2004). In the case of slow CMEs, the situation is opposite. Here, the solar wind pushes the ICME from behind, and since it is a kind of obstacle for the wind flow, the magnetic field lines are deformed in such a way that the resulting tension acts in west direction (so-called "westward deflection"). ICMEs that move at the solar-wind speed are not deflected. Causes for both the eastward and westward deflections are sketched in Figures $1 \mathrm{a}$ and $1 \mathrm{~b}$, respectively. For empirical/statistical confirmation of eastward deflection of fast ICMEs see Figure 6 in Manchester et al. (2017) and Figure 10 in Sudar et al. (2016).

Another important effect is related to the orientation of the poloidal component of the flux-rope magnetic field relative to the ambient heliospheric field. This was first studied by
Vandas et al. (1995, 1996). They considered two different orientations of the flux-rope axis with respect to the ecliptic plane (parallel or perpendicular to it), and found out that radial propagation does not depend much on this orientation. However, it was found that in all cases the rope is deflected to the direction where the poloidal field of the flux rope is opposite from that of the ambient field, i.e., where the reconnection occurs.

The described reconnection process results in one more effect, known as "flux-rope erosion" (see Sect. 7.2 in Manchester et al., 2017, and references therein; see also Sect. 4.2 in Vršnak, 2019). Namely, such reconnection "peels-off" the outer layers of the flux rope. This can significantly affect the ICME propagation. First, the flux rope may lose a considerable fraction of its magnetic flux (e.g., Vršnak, 2019). Second, a significant fraction of the flux-rope mass is lost, which increases the drag parameter $\gamma$ and thus, makes the drag more efficient (see the definition of $\gamma$ below equation (1).

In addition to these systematic global-scale deflections, a CME, no matter fast or slow, can be deflected already in the low corona (e.g., Möstl et al., 2015, and references therein). Such deflections are caused primarily due to the CME interaction with the local ambient magnetic field that sometimes results in sidewise directed magnetic tension and/or magnetic pressure gradients. Such non-radial deflections, as well as the mentioned global-scale ones, can significantly alter the ICME transit times to a certain object in the heliosphere and can introduce an error into predicted arrival times if not taken into account in calculations. Moreover, and certainly the most important consequence of deflections, is that they can lead to the false positive (or negative) prediction of CMEs hitting the Earth.

In the solar-maximum phase, when the CME eruptions are frequent, it happens quite often that two or more CMEs are launched in a close succession from the same or neighbouring source region. Since they move in a more or less the same direction, in the case the preceding CME is slower than the following one, the faster one will catch-up the leading one (e.g., Farrugia \& Berdichevsky, 2004; Shen et al., 2012; Temmer et al., 2012, 2014; Maričić et al., 2014; Rollett et al., 2014, and references therein). After the leading edge of the later CME comes in contact with the rear of the first one, an MHD interaction will start, leading to a deformation of their cross sections, and what is more important, to transfer of momentum from the faster to the slower one. Consequently, the leading CME will be accelerated, whereas the later one will be decelerated, until both getting the same speed, after which they travel "glued" together. Signatures of such merging process can be found in complex ICME structures in the in situ measurements (e.g., Farrugia \& Berdichevsky, 2004; Maričić et al., 2014). In some events, if the magnetic field of the two ICMEs are oriented appropriately, magnetic reconnection of their fields can occur, forming a common magnetic field envelope. Note that if two interacting ICMEs are not moving in the same direction, the interaction causes a deflection of both in opposite directions, as expected from the momentum conservation law (e.g., Rollett et al., 2014; Temmer et al., 2014). Finally, it should be emphasized that CMEs cannot be treated as rigid objects, as they are fluid objects characterized by expansion. Furthermore, CMEs are not coherent MHD structures, as in many instances CME expansion is faster than the local Alfvén speed, so an interaction on a given CME segment will never be communicated to its 
distant segments and the CME shape will be deformed by the interaction (e.g., Savani et al., 2010; Temmer et al., 2014; Owens et al., 2017; Ala-Lahti et al., 2020).

Another type of interaction that can significantly affect the motion and shape of ICMEs is their interaction with high-speed streams (HSSs) in the solar wind that originate from coronal holes (e.g., Temmer et al., 2011; Vršnak et al., 2013). Such streams are characterized by speeds up to $800 \mathrm{~km} \mathrm{~s}^{-1}$ i.e., are much faster than the "standard" slow solar wind. In addition, they have much lower density than the slow wind. Consequently, if a fast ICME encounters the HSS, the drag will become weaker, and thus, the deceleration will weaken too (e.g., Temmer et al., 2011). In the case of slow ICMEs, where the solar wind tends to accelerate the erupting structure, the drag-related acceleration will change likewise. Note that in the latter case, a shock can be formed in the rear of the CME due to a large difference between ICME and HSS speed.

One more important effect is a deformation of the ICME cross section due to the interaction with the ambient plasma. It was noted in some simplified MHD simulations by Cargill et al. (1994, 1996, 2000), as sketched in Figure 2a. Later on, the effect was confirmed also by various heliospheric MHD simulations as reported by, e.g., Vandas et al. (1995) and Owens et al. (2006) and called "pancaking" (see Sect. 9.1 in Manchester et al., 2017, and references therein), as the lateral extent of the erupting flux rope increases ("over-expansion"), and dominates over a somewhat reduced radial expansion (Fig. 2b). This leads to an apparent thinning of the structure, especially compared to the self-similar expansion form, where the structure expands, but without changing its shape. The described effect is caused by vortex motions in the rear of the flux rope (Fig. 2a) caused by its fast motion relative to the ambient magnetoplasma. The increased "inward" pressure gradient at the frontal part causes an increase of the outward turbulenceassociated dynamical pressure at the rear part (like in the case of the airplane wing), leading to the flux-rope flattening. Signatures of pancaking are confirmed by observations of ICMEs by heliospheric imagers that can follow ICMEs from the corona up to distances beyond $1 \mathrm{AU}$, as well as by in situ measurements (e.g., Gulisano et al., 2010; Savani et al., 2010, 2011).

To conclude, the pancaking behaviour is completely different from that of the rain droplets that form a tear-like shape. It is interesting to note that one can find the described effect in the case of cars that have the carrosseries (the carriage body of cars) with the backside "cut" vertically. The effect of the rear turbulence, and the resulting forward directed dynamical-pressure gradient one can see in the fact that there can be more raindrops on the rear car window than on the forward one. Thus, the effect of the ICME "pancaking" is twofold - the increasing angular extent of the ICME, i.e., over-expanding $A$, tends to increase the drag, whereas rear vortices tend to reduce it.

\subsection{CME-driven shocks}

Impulsively accelerated CMEs generate large amplitude fast-MHD waves that can steepen into shocks already in the low corona (e.g., Warmuth, 2015; Vršnak et al., 2016, and references therein). These large amplitude waves and shocks are evidenced by two relevant signatures (e.g., Warmuth, 2007, 2015, and references therein). In close association with the CME main acceleration phase (e.g., Vršnak \& Cliver, 2008,
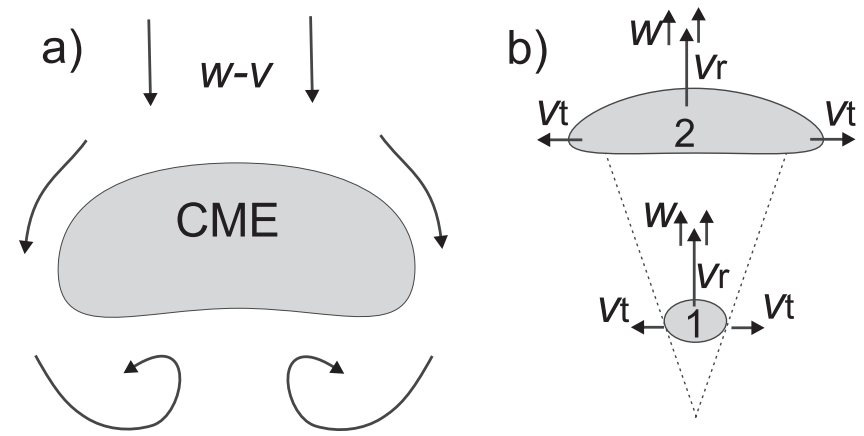

Fig. 2. Pancaking effect. a) Sketch of ambient plasma flow around the CME in the reference frame moving with the flux rope, so the ambient plasma flow has the velocity $w-v$. Vortices behind the CME are indicated b) Rest-frame evolution of the ICME shape (gray shaded) from the heliocentric distance 1 to 2 . Solar wind speed is denoted as $w$, ICME radial speed as $v_{\mathrm{r}}$, and tangential speed as $v_{\mathrm{t}}$. The two dotted lines represent the ICME angular width at position 1, to emphasize the over-expansion.

and references therein), the so-called solar type II radio bursts are frequently recorded in the dynamic radio spectra. In such spectra, where the emission frequency is shown versus time, type II bursts appear as narrow-band emission, slowly drifting from high to low frequencies (Fig. 3a), with drift-rates corresponding to a disturbance that propagates outward at the magnetosonic speed and can be generated only in shocks. Another signature, clearly visualizing the coronal propagation of a large-amplitude wave, are so-called EUV waves or previously EIT waves (Thompson et al., 1998; Warmuth et al., 2001, 2004; Veronig et al., 2008, 2010, 2011; Muhr et al., 2010) revealed by the EUV observations of low corona (Fig. 3b). It should be also mentioned here that in some cases, actually the full 3D coronal shock-wave dome can be inferred or directly observed in EUV images (Vršnak et al., 2006; Veronig et al., 2010; Kozarev et al., 2011, see Fig. 3b). Associated with strong coronal shocks, the so-called Moreton waves (Moreton \& Ramsey, 1960) are sometimes observed in chromospheric spectral lines, associated with type II bursts and EUV waves (Vršnak et al., 2005a). They represent the chromospheric response to the passage of the coronal shock (e.g., Uchida et al., 1973; Vršnak et al., 2002, 2016; Warmuth, 2015; Warmuth et al., 2004; Long et al., 2017, and references therein). Here, it should be mentioned that CMEs can generate also propagating "wave-like" features that are not waves but are either a result of coronal restructuring or a signature of the outer CME shell expansion (e.g., Delannée, 2000; Chen et al., 2005; Attrill et al., 2007, and references therein; for a review on physics of coronal waves and "non-waves" see Long et al., 2017).

Coronal large-amplitude MHD waves are caused by two effects (e.g., Warmuth, 2015; Vršnak et al., 2016, and references therein). First, there is impulsively accelerated upward motion of the CME, and second, there is the so-called over-expansion (e.g., Kienreich et al., 2009; Patsourakos et al., 2010; Patsourakos \& Vourlidas, 2012; Vršnak et al., 2016; Veronig et al., 2018, and references therein) of the CME in the early stage of the eruption. The former effect tends to create a perturbation of the bow-shock type, whereas the latter acts as a 3-dimensional piston (e.g., Vršnak et al., 2016). Note that in 

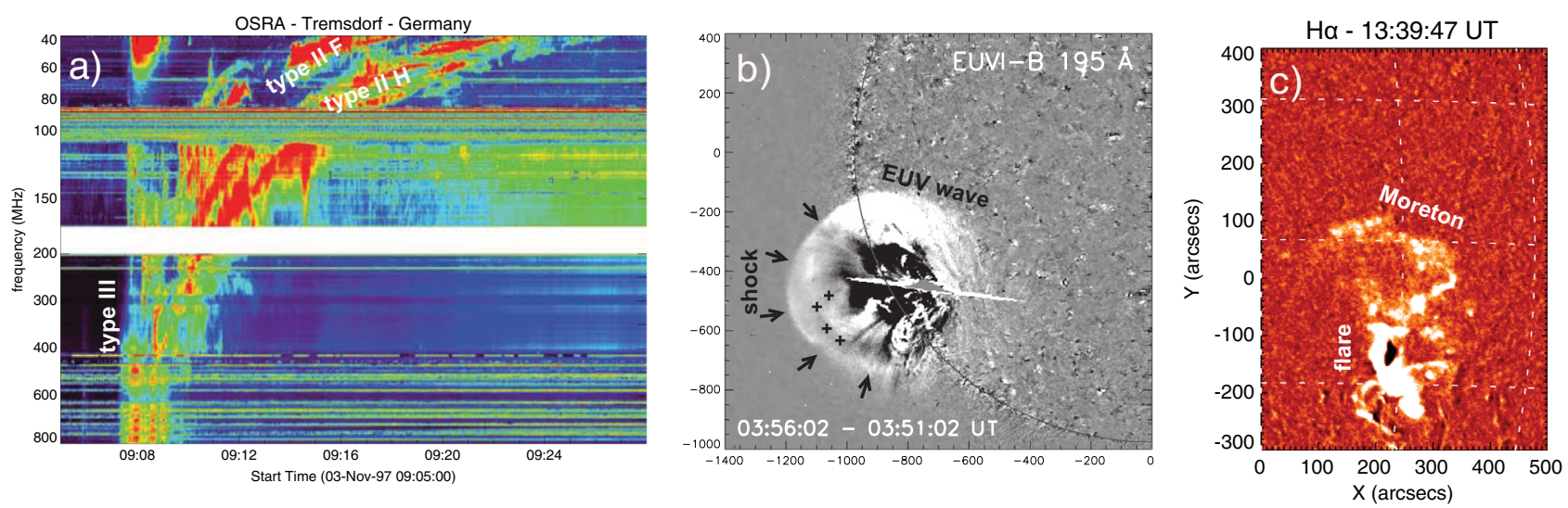

Fig. 3. a) Solar type II radio burst recorded on November 3, 1997, by the OSRA radiospectrograph of the Astrophysikalishes Institut Potsdam at Tremsdorf. Fundamental and harmonic emission bands are indicated (type II F and type II H, respectively). Most often, the onset of type II burst is tightly related to the type III burst. Note that at the y-axis of the spectrum frequencies are presented in the reverse order to give a better impression of disturbance propagating outwars. b) Early stage of the eruption of January 17, 2010, recorded by EUVI-B instrument onboard STEREO-B mission. The coronal shock front is indicated by arrows, the expanding driver by black pluses. The on-disc signature of the shock is marked as EUV wave (for details see Veronig et al., 2010). c) Moreton wave recorded by the Sonnenobservatorium Kanzelhöhe (University of Graz) on May 2, 1998 (for details see Warmuth et al., 2001). The associated flare, usually being also the source of a CME, is also indicated.

the piston case, the source does not have to be supersonic, as the shock is created by non-linear evolution of the large-amplitude wave, i.e., due to the fact that wave elements of higher amplitude have larger phase speed. Thus, since the wave crest moves faster than leading edge of the wave, the wave profile gradually steepens, and eventually becomes a discontinuity, i.e., the MHD shock forms (Vršnak \& Lulić, 2000).

When the outward propagation dominates over the overexpansion, the CME creates only a coronal signature, i.e., the radio type II burst, excited at the CME tip or flanks relatively closely to the tip. In low corona, EUV signatures cannot be found Vršnak et al., 2016). On the other hand, when there is significant impulsive over-expansion, the CME creates the EUVwave signature, and if the shock is strong enough, it creates also an observable chromospheric response in the form of Moreton wave (Vršnak et al., 2016).

CMEs that are powerful enough continue to drive shocks through the upper corona and the interplanetary space. The shock is again a combination of the bow-shock type and the piston-shock type, as CMEs generally expand laterally also in the interplanetary space. In the radio dynamical spectrum we observe them as the so-called decametric, hectometric, and kilometric type II bursts (e.g., Gopalswamy et al., 2000; Vršnak et al., 2001; Gopalswamy, 2006). Clear shock signatures are also recorded ahead of CMEs by the in situ measurements in the vicinity of the Earth (e.g., Dulk et al., 1999; Witasse et al., 2017), and beyond. Here we have two different situations. Sometimes, the shock is attached to the CME like a bow shock (the wave-associated flow speed increases from the shock to the driver). On the other hand, if the CME does not contain enough energy to drive the shock anymore, i.e., it "gets tired" due to a transfer of the momentum to the ambient plasma, the shock detaches from the CME front and continues to travel as a freely-propagating wave (Warmuth, 2015, and references therein). In such a case, the shock-associated flow speed decreases from the shock to the CME. This is possible as the dissipative losses are relatively weak, as well as due to the fact that the ambient density falls-off as $1 / r^{2}$, which compensates the $r^{2}$ increase of the shock area, which enables the preservation of the wave amplitude. Note that the amplitude can even increase due to decreasing Alfvén speed in the interplanetary space.

It should also be noted that due to the continuous expansion of the CME body relative to the solar wind, the so-called reverse shock is sometimes formed at the rear of the ICME. Such a signature can be clearly seen in the events characterized by strong expansion.

Finally, let us mention an important aspect of the presence of the ICME driven shocks. The sheath region between the shock and the driving ICME body is characterized by strong MHD turbulence, so there are always segments of the sheath where at least one magnetic field component of the incoming solar wind is opposite to that of the Earth magnetic field, enabling the component reconnection. Consequently, the shock-driving ICMEs are always geoeffective to a certain degree, even if the magnetic field of the ICME body itself does not have the magnetic field orientation suitable for reconnection with the geomagnetic field.

\subsection{Geoeffectiveness of ICMEs}

ICMEs frequently cause disturbances of the geomagnetic field, sometimes even the most severe ones (e.g., Kilpua et al., 2017; Riley et al., 2018). Generally, the geomagnetic field disturbances, the so-called geomagnetic storms and substorms are described by various "global" indices, such as $K p, a p, a a$, $A m, A E, P C$, Dst, etc. Storms are related to reconnection of interplanetary magnetic field (IMF) and the geomagnetic field at the day-side ("nose reconnection"). They affect significantly whole magnetosphere, from the equator to the poles. Substorms are caused by reconnection in the Earth's magnetic tail, affecting more severely the polar regions. Note that particularly strong geomagnetic effects are related to CMEs occurring in 
close succession, and interacting, either close to Sun or in the interplanetary space (e.g., Liu et al., 2014; Vennerstrom et al., 2016; Lugaz et al., 2017; Riley et al., 2018; Rodrguez Gómez et al., 2020).

The Dst index describes changes of the horizontal field components around the magnetic equator and is based on measurements of several terrestrial magnetometer stations. In the initial phase of the storm, the surface magnetic field is somewhat enhanced, due to the compression caused by increased dynamical pressure of the ICME. Then, after the reconnection of the southward component of the ICME's magnetic field with the northward geomagnetic field component starts, the Dst index reveals a significant decrease of the surface field, with amplitudes sometimes reaching several hundreds of nT, up to almost 600 nT (e.g., Baker et al., 2013). This effect is caused by the enhancement of the so-called ring current (e.g., Sandhu et al., 2018), which is oriented in such a way that it reduces the surface magnetic field. The increase of the ring current is caused by the injection of energized particles into the magnetosphere. It affects not only the magnetosphere, but also the ionosphere and induces geomagnetically induced currents (GICs) on the Earth surface that can cause severe consequences in everyday life (e.g. Feynman \& Gabriel, 2000; Schrijver et al., 2015). Note that although the changes of the Earth's surface field $(\delta B<600 \mathrm{nT})$ are relatively small compared to the strength of the quiet surface field (say, $\approx 40 \mu \mathrm{T}$ at the equator, i.e., the perturbation in strong storms is about $1 \%$ of the equatorial field), they can induce quite strong electric fields and thus, quite strong GICs, due to very fast changes of surface field $(E \propto \mathrm{d} B /$ $\mathrm{d} t)$. Finally, it should be noted that various estimates of the super-storm of 1859 ("Carrington event") indicate that the Dst amplitude might have been larger than $1000 \mathrm{nT}$ (e.g., Siscoe et al., 2006; Cliver \& Dietrich, 2013; Moriña et al., 2019, for a general overview on extreme space weather events see Riley et al., 2018, and references therein).

\section{Space weather forecasting tools}

There is a number of statistical, analytical, and numerical ICME-related forecasting tools. Mainly, they are oriented to the prediction of the arrival time and impact speed of the ICMEs at the Earth (see, e.g., Vršnak et al., 2014; Verbeke et al., 2019; Vourlidas et al., 2019, and references therein). A prediction of the geoeffectiveness of an ICME is a much more demanding problem, because it is very difficult to estimate from the solar CME observations what the southward component of the ICME magnetic field will be when it arrives to the Earth. Hereafter, let us focus on some empirical/statistical and analytical models that are frequently used to predict the arrival and impact speed of a given CME. For a recent overview of active methods see Riley et al. (2018), Verbeke et al. (2019), and Section 5.2 in the review by Manchester et al. (2017).

\subsection{Empirical/statistical tools}

The simplest possible forecasting estimate of the ICME arrival time is based on the so-called "Bruekner $80 \mathrm{~h}$ rule" (Brueckner et al., 1998). It states that the majority of solar eruptions on average will arrive to the Earth in $80 \mathrm{~h}$. Interestingly, this is a quite effective prediction since the vast majority of ICMEs move at speeds close to the solar wind speed or a bit faster, and that is the time for a disturbance moving at $\approx 500 \mathrm{~km} \mathrm{~s}^{-1}$ to come from the Sun to the Earth. Yet, such a prediction fails in the case of slow CMEs, where the transit time can be up to 5-6 days, and especially for the fast ones, which in extreme cases can arrive in less than one day, down to $17.5 \mathrm{~h}$ in the historic Carrington event of September 1, 1859 (Gopalswamy et al., 2005). The latter is especially large drawback, as fast ICMEs tend to be, on average, more geoeffective than the slow or mid-speed ICMEs (e.g., Dumbović et al., 2015, and the references therein).

The mentioned shortcoming regarding very fast and very slow ICMEs can be at least partly eliminated by using the approximation of a "ballistic propagation". In this approximation, the CME speed measured by coronagraphs is used to extrapolate its motion through the heliosphere by assuming that the CME/ICME speed remains roughly constant. This approach is frequently used to connect eruptions observed in the corona with the ICME signatures recorded in situ (e.g., Murray et al., 2018). Again, the problem is that the deceleration of fast ICMEs is neglected, which can cause a wrong CMI-ICME pairing, especially in the phase of high solar activity.

Somewhat more advanced is the empirical, statistics-based, kinematical model by Gopalswamy et al. (2001); see also Reiner et al. (2007). The method is based on the assumption that after the stage of decelerated motion of fast CMEs (or acceleration of slow ones), the ICME enters the regime of constant speed. In the non-constant speed stage, the ICME propagation is approximated by an uniformly decelerating/accelerating motion, where the amount of deceleration/acceleration depends on the ICME speed. This stage is considered to end at heliocentric distances between $\approx 50$ and $\approx 150$ solar radii (see Sect. 5.1 in Manchester et al., 2017, and references therein). The main problem in the real-time forecasting based on this method lies in the fact that the distance at which the CME enters the approximately constant-velocity stage is not known, and it can largely vary from one event to another (for details see Manchester et al., 2017). Furthermore, it does not account for difference in the solar wind conditions from solar minimum to maximum, nor for the ICME-ICME interactions and ICME-HSS interactions. However, the uncertainty in the real velocity of CMEs, as we directly observe only the plane-of-sky component of velocities, remains the most significant drawback of this model.

Let us mention here also the forecasting methods based on the "neural network approach". Such a method was applied by, e.g., Sudar et al. (2016), but the accuracy of the arrival-time prediction was no better than with other empirical/statistical procedures. Such an approach was also tried for forecasting the geoeffectiveness of CMEs (e.g., Valach et al., 2009; Uwamahoro et al., 2012; Dumbović et al., 2015) and Forbush decrease predictions (Dumbovic et al., 2016).

Finally, it should be noted that there is a number of other empirical/statistical methods not mentioned herein. For other examples of various forecasting options see Section 5.2 in Manchester et al. (2017).

\subsection{Analytical models}

Hereafter, two analytical, physics-based CME/ICME propagation models that can be used as forecasting tools are presented. These are the "snow plough" model and the drag-based 
model [for the physical background of these two approaches see, e.g., Tappin, 2006. Both are characterized by a smooth and continuous non-constant acceleration, decreasing from high to low values. Furthermore, in both the acceleration depends on the CME/ICME speed relative to the solar wind speed squared. Although they are both considering the interaction with the ambient plasma, and are both described by similar equations, they are based on different physical principles. The snowplough model (SPM) takes as the basic physical principle only the momentum conservation, whereas the drag-based model (DBM; Vršnak et al., 2013) considers also the MHD nature of the interaction between the CME/ICME and the ambient magnetoplasma.

The SPM is based solely on the momentum-conservation principle (e.g., Feng et al., 2015). In the model it is ad hoc assumed that the plasma ahead of a fast ejection continuously piles up. Let us consider that at a given moment the erupting structure propagates at the instantaneous speed, $V$, relative to the solar wind, i.e., $V=v-w$, where $v$ is the rest-frame speed of the ejection and $w$ is the speed of the ambient plasma. In the absence of external forces, the momentum $M_{\mathrm{tot}} V$ has to remain constant, where $M_{\mathrm{tot}}$ is the total instantaneous mass of the ICME, i.e., the mass of the ejection itself $\left(M_{\mathrm{cme}}\right)$, plus the mass of accumulated ambient plasma (i.e., the virtual mass $M_{\mathrm{v}}$, so $\left.M_{\mathrm{tot}}=M_{\mathrm{cme}}+M_{\mathrm{v}}\right), M_{\mathrm{tot}} V=$ const., meaning $\mathrm{d}\left(M_{\mathrm{tot}} V\right) / \mathrm{d} t=0$. This implies:

$$
M_{\text {tot }} \frac{\mathrm{d} V}{\mathrm{~d} t}+V \frac{\mathrm{d} M_{\mathrm{v}}}{\mathrm{d} t}=0,
$$

where it was assumed $M_{\mathrm{cme}} \approx$ const. Thus, the deceleration reads $\mathrm{d} V / \mathrm{d} t=a=-V\left(\mathrm{~d} M_{\mathrm{v}} / \mathrm{d} t\right) / M_{\text {tot. }}$ On the other hand, the change-rate of the accumulated mass can be expressed as:

$$
\frac{\mathrm{d} M_{\mathrm{v}}}{\mathrm{d} t}=A \rho_{\mathrm{w}} V,
$$

where $\rho_{\mathrm{w}}$ is the ambient density and $A$ is the cross-section area of the ejection. This expression can be rewritten using the sector density $\sigma$ (i.e., the mass per unit solid angle per unit radial distance and the heliocentric solid angle of the ejection, $\Omega$, meaning $A \rho_{\mathrm{w}}=\sigma \Omega$ (Tappin, 2006). Combining equations (2) and (3) one finds the equation of motion:

$$
\frac{\mathrm{d}^{2} r}{\mathrm{~d} t^{2}}=-\frac{A \rho_{\mathrm{w}}}{M_{\mathrm{tot}}}\left(\frac{\mathrm{d} r}{\mathrm{~d} t}-w\right)^{2},
$$

where it was assumed $w=$ const. Since it is taken that $V$ is always positive, i.e., $v>w$, the mass is continuously accumulated (for observations see, e.g., Colaninno \& Vourlidas, 2009; Bein et al., 2013; Feng et al., 2015). Note also that in deriving equation (4) it was assumed that no plasma is escaping sidewise at the ejection flanks, thus the total mass continuously increases. Note that equation (4) shows that the acceleration $a$, has a quadratic dependence on $V$.

Obviously, equation (4) implies that the SPM can be applied only to fast CMEs. In this respect the DBM (Eq. (1)) is better, as it applies also to slow CMEs. The DBM is based on the fact that the ambient plasma is magnetized, so the momentum is transported to the ambient plasma (or from the ambient solar wind to slow ICMEs) by perturbations of the magnetoplasma, i.e., by collisionless magnetosonic waves. In this way, it also explains the formation of CME/ICME-driven shocks, often observed in the case of fast CMEs. Namely, as, e.g., a fast
CME moves through the ambient solar wind, it creates a frontal compression of the ambient magnetoplasma, and the signals about changes of the CME velocity propagate outwards at a magnetosonic speed (Lulić et al., 2013; Vršnak et al., 2016). Due to the non-linear evolution of such a magnetosonic perturbation, the wave crest steepens until it eventually becomes a discontinuity, i.e., the shock (Vršnak \& Lulić, 2000; Vršnak et al., 2016). The equation of motion used in DBM is given by equation (1), presented and discussed in Section 2.3.

The ICME arrival time and impact speed predictions based on DBM are available as a free online forecasting tool that can be found at http://oh.geof.unizg.hr/DBM/dbm.php. It is included also in The Space Situational Awareness (SSA) webpage of ESA (https://swe.ssa.esa.int/sco_pla), and at the NASA scoreboard (https://kauai.ccmc.gsfc.nasa.gov/CMEscoreboard/). Typically, the predictions of the ICME arrival time are at an accuracy of about $\pm 10 \mathrm{~h}$, which is very similar to the more advanced numerical methods. In direct comparison of the DBM and the numerical model ENLIL (for the ENLIL model see, e.g., Odstrcil \& Pizzo, 1999), done for a sample of CMEs/ICMEs, Vršnak et al. (2014) found a very good match between the two. The correspondence was better in periods of low activity than in high-activity phases, mainly due to the fact that ENLIL can track the changes in the ambient solar wind configuration caused by preceding ICMEs. For a detailed comparison of behaviour of a single event see, e.g., Falkenberg et al. (2011). Note that the DBM accounts for the flattening of the frontal edge of fast CMEs, but cannot give the information about ICME pancaking, i.e., lateral over-expansion of the ICME body.

There are various causes that lead to uncertainties in the DBM estimates of the ICME arrival time, impact speed and probability of impact (e.g., Kay \& Gopalswamy, 2018, and references therein). Like in any other forecasting model, first of all, it is the uncertainty in the measured CME speed at the beginning of its heliospheric propagation. This is related not only to the accuracy of measurements themselves, but also on the assumed geometry of the CME leading edge (see, e.g., Žic et al., 2015; Rollett et al., 2016, and references therein) and the applied reduction technique (for an overview see Barnard et al., 2017).

Second, the solar wind speed is basically taken as an average value calculated over a certain period overlapping with the eruption onset, and this can be quite different from the actual one. Finally, the value of the drag parameter $\gamma$ is not well known, primarily due to the unknown drag coefficient $c_{\mathrm{d}}$ (it is usually taken as $c_{\mathrm{d}}=1$ ) and the quite large uncertainty in the mass estimates based on the coronographic observations (Vourlidas et al., 2000; de Koning, 2017). Thus, it is reasonable to broaden the standard DBM (Vršnak et al., 2013) to an ensemble type of modelling, which was performed by Dumbović et al. (2018), who developed the Drag-Based Ensemble Model (DBEM), available as an online tool at the Space Situational Awareness (SSA) European Space Agency (ESA) portal (https://swe.ssa.esa.int/heliospheric-weather). This option of DBEM, i.e., the ensemble approach, with some significant additional improvements (e.g., Kay et al., 2020), is more and more in use as it gives an insight into the uncertainty of the estimate of the mentioned impact parameters. Note that the ensemble modelling is nowadays used also in the case of MHD-simulation forecasting models (e.g., Mays et al., 2015). 
Finally, it should be noted that recently an extended version of DBEM operative tool was developed (DBEMv3) at the Hvar Observatory (Croatia) in collaboration with Institute of Physics of the University of Graz (Austria). This new version allows also the implementation of CME input parameters based on the Graduated Cylindrical Shell technique (GCS; Thernisien et al., 2006, 2009; Thernisien, 2011). The tool is available at https://swe.ssa.esa.int/heliospheric-weather and https://swe.ssa. esa.int/graz-dbem-federated (Čalogović et al., 2021).

\section{Summary and conclusion}

In the present paper, the physical background of the origin, initiation, acceleration, heliospheric propagation and geoeffectiveness of coronal mass ejections is discussed. These general considerations are eventually applied to the space weather forecasting based on empirical/statistical and analytical methods.

Driven by subphotospheric convective motions as well as the photospheric and chromospheric flows, the coronal magnetic field configuration evolves so that electric currents continuously build up, i.e., more and more free magnetic energy and helicity are stored in the system. This process is particularly conspicuous in the dynamically developing magnetic concentrations, such as active regions.

As this process cannot go on endlessly, at a certain critical point, a given "overloaded" coronal structure loses its equilibrium, enters into an unstable state and eventually erupts. The transition to the unstable state can happen quite gradually, over the hours, even days, and the evolution can be described as a series of quasi-equilibrium states. In this process the preeruptive structures usually swell and rise slowly, until reaching a critical height when they lose their equilibrium and erupt. However, sometimes the structure can have a transition to an unstable state relatively rapidly, e.g., when the structure develops fast, like in pre-eruptive restructuring caused by confined impulsive flares, or emergence of an already unstable twisted field.

After the pre-eruptive structure loses its equilibrium, it starts to accelerate upwards due to unbalanced Lorentz force, which can drive the structure to speeds of, say, from $<100$ to $>2000 \mathrm{~km} \mathrm{~s}^{-1}$. In some cases, when the ambient conditions, e.g., when the overlying field is too strong, the eruption is halted at some height. If it finds some upper equilibrium state it stays there, and if not it returns back to a state similar to the pre-eruptive state. We call such events the "failed eruptions". If the eruption manages to overpower all possible obstacles, the coronal coccon becomes a beautiful swaying butterfly winging into the solar wind.

Now, slow butterflies fly carried by the wind, moving faster and faster until they start moving together with the wind. Stronger and more stubborn ones, which try to fly faster than the wind stream, have to fight against the drag, and as they get tired they start to slow down. That is, slow CMEs accelerate, the fast ones decelerate.

Most of empirical and physics-based analytical forecasting methods are based on this effect. In principle, this should work well. However, due to inaccuracies in the initial conditions taken from observations, as well as due to various known and unknown processes occurring during the CME/ICME heliospheric propagation, the accuracy of the $1 \mathrm{AU}$ arrival-time predictions is on average no better than $\pm 10 \mathrm{~h}$, sometimes being as large as 1 day. Yet, in the majority of cases the prediction errors are grouped about $0 \mathrm{~h}$ (for a very detailed review on this topic see Vourlidas et al., 2019, and references therein). Although this may seem very good, and at this stage of space weather forecasting no worse than that provided by very advanced full-MHD simulations, it should be emphasized that empirical and analytic methods are still burdened with a large fraction of too wrong predictions, including the false alarms and missing hits, which will be unavoidable obstacle in their further applications. For now, they have advantage of being very fast, providing instantaneous adjustment to the incoming refreshed observational data. Thus, it seems that the future of the space weather forecasting lies in "physics-based" numerical simulations, which should work well even now, if a more accurate observational input for the initial and boundary conditions would be more accurate. As far as I can see, without better observational input, there will be no significant progress in the field of space weather forecasting, whatsoever we do in advancing the models.

Acknowledgements. This work has been supported by Croatian Science Foundation under the project 7549 "Millimeter and submillimeter observations of the solar chromosphere with ALMA". I'm grateful to the International Space Science Instiute (ISSI) in Bern for hosting the ISSI workshop "The Scientific Foundation of Space Weather", 27 June - 1 July 2016 (conveners: D. Baker, Uni. Colorado; A. Balog, Imperial College; T. Gombosi, Uni. Michigan; A. Arbor; H. Koskinen, Univ. Helsinki; R. von Steiger (main convener, ISSI; A. Veronig, Uni. Graz). I would like to thank my colleagues Mateja Dumbović (Hvar Observatory) and Astrid Veronig (Uni. Graz), as well as two anonymous referees, for their help in preparing and improving this paper. Finally, I'm grateful to Jean Lilensten for huge efforts in organising the ESWW and the ESWW medals contest and especially for organising this 10 years anniversary of the JSWSC, along with Anna Belehaki. The editor thanks Peng-Fei Chen and an anonymous reviewer for their assistance in evaluating this paper.

\section{References}

Ala-Lahti M, Ruohotie J, Good S, Kilpua EKJ, Lugaz N. 2020. Spatial coherence of interplanetary coronal mass ejection sheaths at 1 AU. J Geophys Res (Space Phys) 125(9). https://doi.org/ 10.1029/2020JA028002.

Attrill GDR, Harra LK, van Driel-Gesztelyi L, Démoulin P. 2007. Coronal "Wave": Magnetic Footprint of a Coronal Mass Ejection? Astrophys J 656: L101-L104. https://doi.org/10.1086/512854.

Aurass H, Vršnak B, Hofmann A, Rudžjak V. 1999. Flares in sigmoidal coronal structures a case study. Sol Phys 190: 267-293. https://doi.org/10.1023/A:1005261709955.

Baker DN, Li X, Pulkkinen A, Ngwira CM, Mays ML, Galvin AB, Simunac KDC. 2013. A major solar eruptive event in July 2012: Defining extreme space weather scenarios. Space Weather 11(10): 585-591. https://doi.org/10.1002/swe.20097.

Barnard LA, de Koning CA, Scott CJ, Owens MJ, Wilkinson J, Davies JA. 2017. Testing the current paradigm for space weather prediction with heliospheric imagers. Space Weather 15(6): 782 803. https://doi.org/10.1002/2017SW001609. 
Bein BM, Berkebile-Stoiser S, Veronig AM, Temmer M, Muhr N, Kienreich I, Utz D, Vršnak B. 2011. Impulsive acceleration of coronal mass ejections. I. Statistics and Coronal Mass Ejection Source Region Characteristics. Astrophys J 738: 191. https://doi. org/10.1088/0004-637X/738/2/191.

Bein BM, Berkebile-Stoiser S, Veronig AM, Temmer M, Vršnak B. 2012. Impulsive acceleration of coronal mass ejections. II. Relation to soft X-ray flares and filament eruptions. Astrophys $J$ 755: 44. https://doi.org/10.1088/0004-637X/755/1/44.

Bein BM, Temmer M, Vourlidas A, Veronig AM, Utz D. 2013. The height evolution of the "True" Coronal Mass Ejection Mass derived from STEREO COR1 and COR2 Observations. Astrophys $J$ 768(1): 31 . https://doi.org/10.1088/0004-637X/768/1/31.

Bemporad A, Poletto G, Suess ST, Ko Y-K, Schwadron NA, Elliott HA, Raymond JC. 2006. Current sheet evolution in the aftermath of a CME event. Astrophys J 638: 1110-1128. https://doi.org/ 10.1086/497529.

Berkebile-Stoiser S, Veronig AM, Bein BM, Temmer M. 2012. Relation between the coronal mass ejection acceleration and the non-thermal flare characteristics. Astrophys $J$ 753(1): 88 . https://doi.org/10.1088/0004-637X/753/1/88.

Brueckner GE, Delaboudiniere J-P, Howard RA, Paswaters SE, St. Cyr OC, Schwenn R, Lamy P, Simnett GM, Thompson B, Wang D. 1998. Geomagnetic storms caused by coronal mass ejections (CMEs): March 1996 through June 1997. Geophys Res Lett 25: 3019-3022. https://doi.org/10.1029/98GL00704.

Cargill PJ. 2004. On the aerodynamic drag force acting on interplanetary coronal mass ejections. Sol Phys 221: 135-149. https://doi.org/10.1023/B:SOLA.0000033366.10725.a2.

Cargill PJ, Chen J, Spicer DS, Zalesak ST. 1994. The deformation of flux tubes in the solar wind with applications to the structure of magnetic clouds and CMEs. In: Solar Dynamic Phenomena and Solar Wind Consequences, the Third SOHO Workshop. Hunt JJ (Ed.), vol. 373 of ESA Special Publication, pp. 291.

Cargill PJ, Chen J, Spicer DS, Zalesak ST. 1996. Magnetohydrodynamic simulations of the motion of magnetic flux tubes through a magnetized plasma. J Geophys Res 101: 4855-4870. https://doi. org/10.1029/95JA03769.

Cargill PJ, Schmidt J, Spicer DS, Zalesak ST. 2000. Magnetic structure of overexpanding coronal mass ejections: Numerical models. J Geophys Res 105: 7509-7520. https://doi.org/10.1029/ 1999JA900479.

Chen J. 1989. Effects of toroidal forces in current loops embedded in a background plasma. Astrophys J 338: 453-470. https://doi.org/ 10.1086/167211.

Chen PF, Fang C, Shibata K. 2005. A full view of EIT waves. Astrophys J 622: 1202-1210. https://doi.org/10.1086/428084.

Cliver EW, Dietrich WF. 2013. The 1859 space weather event revisited: limits of extreme activity. J Space Weather Space Clim 3: A31. https://doi.org/10.1051/swsc/2013053.

Colaninno RC, Vourlidas A. 2009. First determination of the true mass of coronal mass ejections: A novel approach to using the two STEREO viewpoints. Astrophys $J$ 698(1): 852-858. https://doi. org/10.1088/0004-637X/698/1/852.

Čalogović J, Dumbović M, Sudar D, Vršnak B, Martinić K, Temmer M, Veronig A. 2021. Probabilistic Drag-Based Ensemble Model (DBEM) evaluation for heliospheric propagation of CMEs. Solar Phys. submitted.

de Koning CA. 2017. Lessons learned from the three-view determination of CME Mass. Astrophys J 844(1): 61. https://doi.org/ 10.3847/1538-4357/aa7a09.

Delannée C. 2000. Another view of the EIT wave phenomenon. Astrophys J 545(1): 512-523. https://doi.org/10.1086/317777.
Dulk GA, Leblanc Y, Bougeret J-L. 1999. Type II shock and CME from the corona to 1 AU. Geophys Res Lett 26(15): 2331-2334. https://doi.org/10.1029/1999GL900454.

Dumbović M, Devos A, Vršnak B, Sudar D, Rodriguez L, Ruždjak D, Leer K, Vennerstrøm S, Veronig A. 2015. Geoeffectiveness of coronal mass ejections in the SOHO Era. Sol Phys 290(2): 579 612. https://doi.org/10.1007/s11207-014-0613-8.

Dumbović M, Čalogović J, Vršnak B, Temmer M, Mays ML, Veronig A, Piantschitsch I. 2018. The Drag-based Ensemble Model (DBEM) for Coronal Mass Ejection Propagation. Astrophys $J$ 854(2): 180. https://doi.org/10.3847/1538-4357/aaaa66.

Dumbović M, Vršnak B, Čalogović J. 2016. Forbush decrease prediction based on remote solar observations. Sol Phys 291(1): 285-302. https://doi.org/10.1007/s11207-015-0819-4.

Dumbović M, Vršnak B, Čalogović J, Župan R. 2012. Cosmic ray modulation by different types of solar wind disturbances. Astron Astrophys 538: A28. https://doi.org/10.1051/0004-6361/201117710.

Engvold O. 1988. Prominence Environment. In: Dynamics and Structure of Quiescent Solar Prominences. Astrophysics and Space Science Library, vol 150, Priest ER (Ed.), Springer, Dordrecht. https://doi.org/10.1007/978-94-009-3077-3_3.

Falkenberg TV, Vennerstrom S, Brain DA, Delory G, Taktakishvili A. 2011. Multipoint observations of coronal mass ejection and solar energetic particle events on Mars and Earth during November 2001. J Geophys Res 116: A06104. https://doi.org/10.1029/ 2010JA016279.

Farrugia C, Berdichevsky D. 2004. Evolutionary signatures in complex ejecta and their driven shocks. Ann Geophys 22(10): 3679-3698. https://doi.org/10.5194/angeo-22-3679-2004.

Feng L, Wang Y, Shen F, Shen C, Inhester B, Lu L, Gan W. 2015. Why does the apparent mass of a coronal mass ejection increase? Astrophys J 812(1): 70. https://doi.org/10.1088/0004-637X/812/1/70.

Feynman J, Gabriel SB. 2000. On space weather consequences and predictions. J Geophys Res 105(A5): 10543-10564. https://doi. org/10.1029/1999JA000141.

Garren DA, Chen J. 1994. Lorentz self-forces on curved current loops. Phys Plasmas 1: 3425-3436. https://doi.org/10.1063/ 1.870491.

Gibson SE, Fan Y, Török T, Kliem B. 2006. The evolving sigmoid: Evidence for magnetic flux ropes in the corona before, during, and after CMES. Space Sci Rev 124: 131-144. https://doi.org/10.1007/ s11214-006-9101-2.

Gopalswamy N. 2006. Properties of Interplanetary Coronal Mass Ejections. Space Sci Rev 124: 145-168. https://doi.org/10.1007/ s11214-006-9102-1.

Gopalswamy N, Lara A, Lepping RP, Kaiser ML, Berdichevsky D, St. Cyr OC. 2000. Interplanetary acceleration of coronal mass ejections. Geophys Res Lett 27: 145-148.

Gopalswamy N, Lara A, Yashiro S, Kaiser ML, Howard RA. 2001. Predicting the 1-AU arrival times of coronal mass ejections. J Geophys Res 106: 29207-29218. https://doi.org/10.1029/ 2001JA000177.

Gopalswamy N, Mäkelä P, Xie H, Akiyama S, Yashiro S. 2009. CME interactions with coronal holes and their interplanetary consequences. J Geophys Res 114, A3. https://doi.org/10.1029/ 2008JA013686.

Gopalswamy N, Yashiro S, Liu Y, Michalek G, Vourlidas A, Kaiser ML, Howard RA. 2005. Coronal mass ejections and other extreme characteristics of the 2003 October-November solar eruptions. J Geophys Res 110: 9. https://doi.org/10.1029/2004JA010958.

Green LM, Török T, Vršnak B, Manchester W, Veronig A. 2018. The Origin, Early Evolution and Predictability of Solar Eruptions. Space Sci Rev 214: 46. https://doi.org/10.1007/s11214-017-0462-5. 
Gulisano AM, Démoulin P, Dasso S, Ruiz ME, Marsch E. 2010. Global and local expansion of magnetic clouds in the inner heliosphere. Astron Astrophys 509: A39. https://doi.org/10.1051/ 0004-6361/200912375.

Hood A, Anzer U. 1987. The stability of line tied force-free cylindrical arcades: Is an active region filament a requirement for a two-ribbon flare? Sol Phys 111(2): 333-346. https://doi.org/ 10.1007/BF00148524.

Kay C, Gopalswamy N. 2018. The Effects of Uncertainty in Initial CME Input Parameters on Deflection, Rotation, $\mathrm{B}_{\mathrm{z}}$, and Arrival Time Predictions. J Geophys Res (Space Phys) 123(9): 72207240. https://doi.org/10.1029/2018JA025780.

Kay C, Mays ML, Verbeke C. 2020. Identifying Critical Input Parameters for Improving Drag-Based CME Arrival Time Predictions. Space Weather 18(1): e02382. https://doi.org/10.1029/ 2019SW002382.

Kienreich IW, Temmer M, Veronig AM. 2009. STEREO quadrature observations of the three-dimensional structure and driver of a global coronal wave. Astrophys J Lett 703(2): L118-L122. https://doi.org/10.1088/0004-637X/703/2/L118.

Kilpua EKJ, Balogh A, von Steiger R, Liu YD. 2017. Geoeffective Properties of Solar Transients and Stream Interaction Regions. Space Sci Rev 212(3-4): 1271-1314. https://doi.org/10.1007/ s11214-017-0411-3.

Knipp DJ, Tobiska WK, Emery BA. 2004. Direct and indirect thermospheric heating sources for solar cycles 21-23. Sol Phys 224(1-2): 495-505. https://doi.org/10.1007/s11207-005-6393-4.

Ko Y-K, Raymond JC, Lin J, Lawrence G, Li J, Fludra A. 2003. Dynamical and physical properties of a post-coronal mass ejection current sheet. Astrophys J 594: 1068-1084. https://doi.org/ 10.1086/376982.

Koskinen HEJ, Baker DN, Balogh A, Gombosi T, Veronig A, von Steiger R. 2017. Achievements and challenges in the science of space weather. Space Sci Rev 212(3-4): 1137-1157. https://doi. org/10.1007/s11214-017-0390-4.

Kozarev KA, Korreck KE, Lobzin VV, Weber MA, Schwadron NA. 2011. Off-limb Solar Coronal Wavefronts from SDO/AIA Extreme-ultraviolet Observations - Implications for Particle Production. Astrophys J Lett 733(2): L25. https://doi.org/10.1088/ 2041-8205/733/2/L25.

Krauss S, Temmer M, Veronig A, Baur O, Lammer H. 2015. Thermospheric and geomagnetic responses to interplanetary coronal mass ejections observed by ACE and GRACE: Statistical results. J Geophys Res (Space Phys) 120(10): 8848-8860. https://doi.org/10.1002/2015JA021702.

Liu YD, Luhmann JG, Kajdič P, Kilpua EKJ, Lugaz N, et al. 2014. Observations of an extreme storm in interplanetary space caused by successive coronal mass ejections. Nature Commun 5: 3481. https://doi.org/10.1038/ncomms4481.

Long DM, Bloomfield DS, Chen PF, Downs C, Gallagher PT, et al. et al. 2017. Understanding the physical nature of coronal "EIT Waves". Sol Phys 292(1): 7. https://doi.org/10.1007/s11207-0161030-y.

Lugaz N, Temmer M, Wang Y, Farrugia CJ. 2017. The interaction of successive coronal mass ejections: A review. Sol Phys 292(4): 64. https://doi.org/10.1007/s11207-017-1091-6.

Lulić S, Vršnak B, Žic T, Kienreich IW, Muhr N, Temmer M, Veronig AM. 2013. Formation of coronal shock waves. Sol Phys 286(2): 509-528. https://doi.org/10.1007/s11207-013-0287-7.

Manchester W, Kilpua EKJ, Liu YD, Lugaz N, Riley P, Török T, Vršnak B. 2017. The physical processes of CME/ICME evolution. Space Sci Rev 212(3-4): 1159-1219. https://doi.org/10.1007/ s11214-017-0394-0.
Maričić D, Vršnak B, Dumbović M, Žic T, Roša D, et al. 2014. Kinematics of interacting ICMEs and related forbush decrease: Case study. Sol Phys 289: 351-368. https://doi.org/10.1007/ s11207-013-0314-8.

Maričić D, Vršnak B, Stanger AL, Veronig AM, Temmer M, Roša D. 2007. Acceleration phase of coronal mass ejections: II. Synchronization of the energy release in the associated flare. Sol Phys 241: 99-112. https://doi.org/10.1007/s11207-007-0291-x.

Mays ML, Taktakishvili A, Pulkkinen A, MacNeice PJ, Rastätter L, et al. 2015. Ensemble modeling of CMEs Using the WSA-ENLIL +Cone Model. Sol Phys 290(6): 1775-1814. https://doi.org/ 10.1007/s11207-015-0692-1.

Miklenic CH, Veronig AM, Vršnak B. 2009. Temporal comparison of nonthermal flare emission and magnetic-flux change rates. Astron Astrophys 499: 893-904. https://doi.org/10.1051/00046361/200810947.

Miklenic CH, Veronig AM, Vršnak B, Hanslmeier A. 2007. Reconnection and energy release rates in a two-ribbon flare. Astron Astrophys 461: 697-706. https://doi.org/10.1051/00046361:20065751.

Moreton GE, Ramsey HE. 1960. Recent observations of dynamical phenomena associated with solar flares. Publ Astron Soc Pac 72: 357.

Moriña D, Serra I, Puig P, Corral Á. 2019. Probability estimation of a Carrington-like geomagnetic storm. Scientific Rep. 9: 2393. https://doi.org/10.1038/s41598-019-38918-8.

Möstl C, Rollett T, Frahm RA, Liu YD, Long DM, et al. 2015. Strong coronal channelling and interplanetary evolution of a solar storm up to Earth and Mars. Nature Commun 6: 7135. https://doi. org/10.1038/ncomms8135.

Muhr N, Vršnak B, Temmer M, Veronig AM, Magdalenić J. 2010. Analysis of a global moreton wave observed on 2003 October 28. Astrophys J 708(2): 1639-1649. https://doi.org/10.1088/0004637X/708/2/1639.

Murray SA, Guerra JA, Zucca P, Park S-H, Carley EP, Gallagher PT, Vilmer N, Bothmer V. 2018. Connecting coronal mass ejections to their solar active region sources: combining results from the HELCATS and FLARECAST projects. Sol Phys 293(4): 60. https://doi.org/10.1007/s11207-018-1287-4.

Nindos A, Patsourakos S, Vourlidas A, Cheng X, Zhang J. 2020. When do solar erupting hot magnetic flux ropes form? Astron Astrophys 642: A109. https://doi.org/10.1051/0004-6361/ 202038832.

Odstrčil D, Pizzo VJ. 1999. Distortion of the interplanetary magnetic field by three-dimensional propagation of coronal mass ejections in a structured solar wind. J Geophys Res 104: 28225-28240. https://doi.org/10.1029/1999JA900319.

Ouyang Y, Zhou YH, Chen PF, Fang C. 2017. Chirality and magnetic configurations of solar filaments. Astrophys J 835(1): 94. https://doi.org/10.3847/1538-4357/835/1/94.

Owens MJ, Lockwood M, Barnard LA. 2017. Coronal mass ejections are not coherent magnetohydrodynamic structures. Scientific Rep 7: 4152. https://doi.org/10.1038/s41598-017-04546-3.

Owens MJ, Merkin VG, Riley P. 2006. A kinematically distorted flux rope model for magnetic clouds. J Geophys Res (Space Phys) 111: A03104. https://doi.org/10.1029/2005JA011460.

Parnell CE, De Moortel I. 2012. A contemporary view of coronal heating. Philos Trans R Soc London Ser A 370(1970): 3217-3240. https://doi.org/10.1098/rsta.2012.0113.

Patsourakos S, Vourlidas A. 2012. On the nature and genesis of EUV Waves: A synthesis of observations from SOHO, STEREO, SDO, and Hinode (Invited Review). Sol Phys 281(1): 187-222. https://doi.org/10.1007/s11207-012-9988-6. 
Patsourakos S, Vourlidas A, Stenborg G. 2010. The genesis of an impulsive coronal mass ejection observed at ultra-high cadence by AIA on SDO. Astrophys J Lett 724(2): L188-L193. https://doi.org/ 10.1088/2041-8205/724/2/L188.

Pulkkinen T. 2007. Space weather: Terrestrial perspective. Living Rev Sol Phys 4(1): 1. https://doi.org/10.12942/lrsp-2007-1.

Qiu J, Yurchyshyn VB. 2005. Magnetic reconnection flux and coronal mass ejection velocity. Astrophys J Lett 634: L121-L124. https://doi.org/10.1086/498716.

Reiner MJ, Kaiser ML, Bougeret J-L. 2007. Coronal and interplanetary propagation of $\mathrm{CME} / \mathrm{shocks}$ from radio, in situ and whitelight observations. Astrophys J 663: 1369-1385. https://doi.org/ 10.1086/518683.

Riley P, Baker D, Liu YD, Verronen P, Singer H, Güdel M. 2018. Extreme space weather events: from cradle to grave. Space Sci Rev 214(1): 21. https://doi.org/10.1007/s11214-017-0456-3.

Rodrguez Gómez JM, Podladchikova T, Veronig A, Ruzmaikin A, Feynman J, Petrukovich A. 2020. Clustering of fast coronal mass ejections during solar cycles 23 and 24 and the implications for CME-CME interactions. Astrophys J 899(1): 47. https://doi.org/ 10.3847/1538-4357/ab9e72.

Rollett T, Möstl C, Isavnin A, Davies JA, Kubicka M, Amerstorfer UV, Harrison RA. 2016. ElEvoHI: A novel CME prediction tool for heliospheric imaging combining an elliptical front with dragbased model fitting. Astrophys J 824(2): 131. https://doi.org/ 10.3847/0004-637X/824/2/131.

Rollett T, Möstl C, Temmer M, Frahm RA, Davies JA, et al. 2014. Combined multipoint remote and in situ observations of the asymmetric evolution of a fast solar coronal mass ejection. Astrophys J 709: L6. https://doi.org/10.1088/2041-8205/790/1/L6.

Sandhu JK, Rae IJ, Freeman MP, Forsyth C, Gkioulidou M, Reeves GD, Spence HE, Jackman CM, Lam MM. 2018. Energization of the ring current by substorms. J Geophys Res (Space Phys) 123(10): 8131-8148. https://doi.org/10.1029/2018JA025766.

Savani NP, Owens MJ, Rouillard AP, Forsyth RJ, Davies JA. 2010. Observational evidence of a coronal mass ejection distortion directly attributable to a structured solar wind. Astrophys $J$ Lett 714(1): L128-L132. https://doi.org/10.1088/2041-8205/714/1/ L128.

Savani NP, Owens MJ, Rouillard AP, Forsyth RJ, Kusano K, Shiota D, Kataoka R, Jian L, Bothmer V. 2011. Evolution of coronal mass ejection morphology with increasing heliocentric distance. II. In Situ Observations. Astrophys J 732(2): 117. https://doi.org/ 10.1088/0004-637X/732/2/117.

Schmieder B, Aulanier G, Vršnak B. 2015. Flare-CME models: An Observational perspective (invited review). Sol Phys 290: 34573486. https://doi.org/10.1007/s11207-015-0712-1.

Schrijver CJ, Kauristie K, Aylward AD, Denardini CM, Gibson SE, et al. 2015. Understanding space weather to shield society: A global road map for 2015-2025 commissioned by COSPAR and ILWS. Adv Space Res 55(12): 2745-2807. https://doi.org/10.1016/ j.asr.2015.03.023.

Schwenn R. 2006. Space weather: The solar perspective. Living Rev Sol Phys 3(1): 2. 10.12942/lrsp-2006-2.

Shen C, Wang Y, Wang S, Liu Y, Liu R, Vourlidas A, Miao B, Ye P, Liu J, Zhou Z. 2012. Super-elastic collision of large-scale magnetized plasmoids in the heliosphere. Nature Phys 8(12): 923-928. https://doi.org/10.1038/nphys2440.

Siscoe G, Crooker NU, Clauer CR. 2006. Dst of the Carrington storm of 1859. Adv Space Res 38(2): 173-179. https://doi.org/10.1016/j. asr.2005.02.102.

Song HQ, Zhang J, Chen Y, Cheng X. 2014. Direct observations of magnetic flux rope formation during a solar coronal mass ejection.
Astrophys J Lett 792(2): L40. https://doi.org/10.1088/2041-8205/ 792/2/L40

Sudar D, Vršnak B, Dumbović M. 2016. Predicting coronal mass ejections transit times to Earth with neural network. Mon Not $R$ Astron Soc 456(2): 1542-1548. https://doi.org/10.1093/mnras/ stv2782.

Tappin SJ. 2006. The Deceleration of an Interplanetary Transient from the Sun to 5 AU. Sol Phys 233: 233-248. https://doi.org/ 10.1007/s11207-006-2065-2.

Temmer M, Rollett T, Möstl C, Veronig AM, Vršnak B, Odstrčil D. 2011. Influence of the ambient solar wind flow on the propagation behavior of interplanetary coronal mass ejections. Astrophys $\mathbf{J} 743$ : 101-112. https://doi.org/10.1088/0004-637X/743/2/101.

Temmer M, Veronig AM, Kontar EP, Krucker S, Vršnak B. 2010. Combined STEREO/RHESSI study of coronal mass ejection acceleration and particle acceleration in solar flares. Astrophys $J$ 712: 1410-1420. https://doi.org/10.1088/0004-637X/712/2/1410.

Temmer M, Veronig AM, Peinhart V, Vršnak B. 2014. Asymmetry in the CME-CME Interaction process for the events from 2011 February 14-15. Astrophys J 785: 85. https://doi.org/10.1088/ 0004-637X/785/2/85.

Temmer M, Veronig AM, Vršnak B, Rybák J., et al. 2008. Acceleration in fast halo CMEs and synchronized flare HXR bursts. Astrophys J 673: L95-L98. https://doi.org/10.1086/527414.

Temmer M, Vršnak B, Rollett T, Bein B, de Koning CA, et al. 2012. Characteristics of kinematics of a coronal mass ejection during the 2010 August 1 CME-CME interaction event. Astrophys J 749: 57. https://doi.org/10.1088/0004-637X/749/1/57.

Thernisien A. 2011. Implementation of the graduated cylindrical shell model for the three-dimensional reconstruction of coronal mass ejections. Astrophys J Suppl Ser 194(2): 33. https://doi.org/ 10.1088/0067-0049/194/2/33.

Thernisien A, Vourlidas A, Howard RA. 2009. Forward modeling of coronal mass ejections using STEREO/SECCHI data. Sol Phys 256(1-2): 111-130. https://doi.org/10.1007/s11207-009-9346-5.

Thernisien AFR, Howard RA, Vourlidas A. 2006. Modeling of flux rope coronal mass ejections. Astrophys J 652(1): 763-773. https://doi.org/10.1086/508254.

Thompson BJ, Plunkett SP, Gurman JB, Newmark JS, St. Cyr OC, Michels DJ. 1998. SOHO/EIT observations of an Earth-directed coronal mass ejection on May 12, 1997. Geophys Res Lett 25: 2465-2468. https://doi.org/10.1029/98GL50429.

Titov VS, Démoulin P. 1999. Basic topology of twisted magnetic configurations in solar flares. Astron Astrophys 351: 707-720.

Török T, Kliem B. 2005. Confined and Ejective Eruptions of Kinkunstable Flux Ropes. Astrophys J Lett 630: L97-L100. https://doi. org/10.1086/462412.

Tschernitz J, Veronig AM, Thalmann JK, Hinterreiter J, Pötzi W. 2018. Reconnection fluxes in eruptive and confined flares and implications for superflares on the Sun. Astrophys $J$ 853(1): 41. https://doi.org/10.3847/1538-4357/aaa199.

Tsurutani BT, Gonzalez WD, Gonzalez ALC, Guarnieri FL, Gopalswamy N, et al. 2006a. Corotating solar wind streams and recurrent geomagnetic activity: A review. J Geophys Res (Space Phys) 111(A7): A07S01. https://doi.org/10.1029/2005JA011273.

Tsurutani BT, Gonzalez WD, Gonzalez ALC, Tang F, Arballo JK, Okada M. 1995. Interplanetary origin of geomagnetic activity in the declining phase of the solar cycle. J Geophys Res 100(A11): 21717-21734. https://doi.org/10.1029/95JA01476.

Tsurutani BT, Lakhina GS, Hajra R. 2020. The physics of space weather/solar-terrestrial physics (STP): what we know now and what the current and future challenges are. Nonlinear Process Geophys 27(1): 75-119. https://doi.org/10.5194/npg-27-75-2020. 
Tsurutani BT, McPherron RL, Gonzalez WD, Lu G, Sobral JHA, Gopalswamy N. 2006b. Introduction to special section on corotating solar wind streams and recurrent geomagnetic activity. J Geophys Res (Space Phys) 111(A7): A07S00. https://doi.org/ 10.1029/2006JA011745.

Uchida Y, Altschuler MD, Newkirk GJ. 1973. Flare-produced coronal MHD-fast-mode wavefronts and Moreton's wave phenomenon. Sol Phys 28: 495. https://doi.org/10.1007/BF00152320.

Uwamahoro J, McKinnell LA, Habarulema JB. 2012. Estimating the geoeffectiveness of halo CMEs from associated solar and IP parameters using neural networks. Ann Geophys 30(6): 963-972. https://doi.org/10.5194/angeo-30-963-2012.

Valach F, Revallo M, Bochnček J, Hejda P. 2009. Solar energetic particle flux enhancement as a predictor of geomagnetic activity in a neural network-based model. Space Weather 7(4): S04004. https://doi.org/10.1029/2008SW000421.

Vandas M, Fischer S, Dryer M, Smith Z, Detman T. 1995. Simulation of magnetic cloud propagation in the inner heliosphere in two-dimensions. 1. A loop perpendicular to the ecliptic plane. J Geophys Res 100(A7): 12285-12292. https://doi.org/10.1029/ 94JA03279.

Vandas M, Fischer S, Dryer M, Smith Z, Detman T. 1996. Simulation of magnetic cloud propagation in the inner heliosphere in two dimensions 2. A loop parallel to the ecliptic plane and the role of helicity. J Geophys Res 101: 2505-2510. https://doi.org/ 10.1029/95JA02446.

Vennerstrom S, Lefevre L, Dumbović M, Crosby N, Malandraki O, et al. 2016. Extreme Geomagnetic Storms - 1868-2010. Sol Phys 291(5): 1447-1481. https://doi.org/10.1007/s11207-016-0897-y.

Verbanac G, Vršnak B, Veronig A, Temmer M. 2011a. Equatorial coronal holes, solar wind high-speed streams, and their geoeffectiveness. Astron Astrophys 526: A20. https://doi.org/10.1051/ 0004-6361/201014617.

Verbeke C, Mays ML, Temmer M, Bingham S, Steenburgh R, et al. 2019. Benchmarking CME arrival time and impact: progress on metadata, metrics, and events. Space Weather 17(1): 6-26. https://doi.org/10.1029/2018SW002046.

Veronig AM, Gömöry P, Kienreich IW, Muhr N, Vršnak B, Temmer M, Warren HP. 2011. Plasma diagnostics of an EIT wave observed by Hinode/EIS and SDO/AIA. Astrophys J Lett 743(1): L10. https://doi.org/10.1088/2041-8205/743/1/L10.

Veronig AM, Karlický M, Vršnak B, Temmer M, Magdalenić J, Dennis BR, Otruba W, Pötzi W. 2006. X-ray sources and magnetic reconnection in the X3.9 flare of 2003 November 3. Astron Astrophys 446: 675-690. https://doi.org/10.1051/00046361:20053112.

Veronig AM, Muhr N, Kienreich IW, Temmer M, Vršnak B. 2010. First Observations of a Dome-shaped Large-scale Coronal Extreme-ultraviolet Wave. Astrophys J Lett 716(1): L57-L62. https://doi.org/10.1088/2041-8205/716/1/L57.

Veronig AM, Podladchikova T, Dissauer K, Temmer M, Seaton DB, Long D, Guo J, Vršnak B, Harra L, Kliem B. 2018. Genesis and impulsive evolution of the 2017 September 10 coronal mass ejection. Astrophys J 868: 107. https://doi.org/10.3847/1538-4357/ aaeac5.

Veronig AM, Temmer M, Vršnak B. 2008. High-cadence observations of a global coronal wave by STEREO EUVI. Astrophys $J$ Lett 681(2): L113. https://doi.org/10.1086/590493.

Vourlidas A, Patsourakos S, Savani NP. 2019. Predicting the geoeffective properties of coronal mass ejections: current status, open issues and path forward. Philos Trans $R$ Soc London Ser A 377(2148): 20180,096. https://doi.org/10.1098/rsta.2018.0096.
Vourlidas A, Subramanian P, Dere KP, Howard RA. 2000. Largeangle spectrometric coronagraph measurements of the energetics of coronal mass ejections. Astrophys $J$ 534: 456-467. https://doi. org/10.1086/308747.

Vršnak B. 1990. Eruptive instability of cylindrical prominences. Sol Phys 129: 295-312. https://doi.org/10.1007/BF00159042.

Vršnak B. 2001. Dynamics of solar coronal eruptions. J Geophys Res 106: 25249-25260. https://doi.org/10.1029/2000JA004007.

Vršnak B. 2008. Processes and mechanisms governing the initiation and propagation of CMEs. Ann Geophys 26: 3089-3101. https://doi.org/10.5194/angeo-26-3089-2008.

Vršnak B. 2009. The Role of reconnection in the CME/Flare process. Adv Geosci 14: 43. ISBN 9789812836,199.

Vršnak B. 2016. Solar eruptions: The CME-flare relationship. Astron Nachr 337: 1002. https://doi.org/10.1002/asna.201612424.

Vršnak B. 2019. Gradual pre-eruptive phase of solar coronal eruptions. Front Astron Space Sci 6: 28. https://doi.org/10.3389/ fspas.2019.00028.

Vršnak B, Aurass H, Magdalenić J, Gopalswamy N. 2001. Bandsplitting of coronal and interplanetary type II bursts. I. Basic properties. Astron Astrophys 377: 321-329. https://doi.org/ 10.1051/0004-6361:20011067.

Vršnak B, Cliver EW. 2008. Origin of coronal shock waves. Invited Review. Sol Phys 253: 215-235. https://doi.org/10.1007/s11207008-9241-5.

Vršnak B, Lulić S. 2000. Formation of coronal MHD shock waves I. The basic mechanism. Sol Phys 196: 157-180. https://doi.org/ 10.1023/A:1005236804727.

Vršnak B, Magdalenić J, Temmer M, Veronig A, Warmuth A, Mann G, Aurass H, Otruba W. 2005a. Broadband metric-range radio emission associated with a Moreton/EIT wave. Astrophys J Lett 625: L67-L70. https://doi.org/10.1086/430763.

Vršnak B, Maričić D, Stanger AL, Veronig A. 2004a. Coronal Mass Ejection of 15 May 2001: II. Coupling of the CME acceleration and the flare energy release. Sol Phys 225: 355-378. https://doi. org/10.1007/s11207-004-4995-x.

Vršnak B, Maričić D, Stanger AL, Veronig AM, Temmer M, Roša D. 2007. Acceleration phase of coronal mass ejections: I. Temporal and spatial scales. Sol Phys 241: 85-98. https://doi. org/10.1007/s11207-006-0290-3.

Vršnak B, Poletto G, Vujić E, Vourlidas A, Ko YK, et al. 2009. Morphology and density structure of post-CME current sheets. Astron Astrophys 499(3): 905-916. https://doi.org/10.1051/00046361/200810844.

Vršnak B, Ruždjak D, Sudar D, Gopalswamy N. 2004b. Kinematics of coronal mass ejections between 2 and 30 solar radii. What can be learned about forces governing the eruption? Astron Astrophys 423: 717-728. https://doi.org/10.1051/0004-6361:20047169.

Vršnak B, Sudar D, Ruždjak D. 2005b. The CME-flare relationship: Are there really two types of CMEs? Astron Astrophys 435: 11491157. https://doi.org/10.1051/0004-6361:20042166.

Vršnak B, Temmer M, Žic T, Taktakishvili A, Dumbović M, Möstl C, Veronig AM, Mays ML, Odstrčil D. 2014. Heliospheric propagation of coronal mass ejections: Comparison of numerical WSAENLIL+Cone model and analytical drag-based model. Astrophys $J$ Suppl Ser 213: 21. https://doi.org/10.1088/0067-0049/213/2/21.

Vršnak B, Žic T, Vrbanec D, Temmer M, Rollett T, et al. 2013. Propagation of interplanetary coronal mass ejections: The dragbased model. Sol Phys 285: 295-315. https://doi.org/10.1007/ s11207-012-0035-4.

Vršnak B, Vrbanec D, Čalogović J. 2008. Dynamics of coronal mass ejections. The mass-scaling of the aerodynamic drag. Astron 
Astrophys 490: 811-815. https://doi.org/10.1051/0004-6361: 200810215.

Vršnak B, Žic T, Lulić S, Temmer M, Veronig AM. 2016. Formation of coronal large-amplitude waves and the chromospheric response. Sol Phys 291(1): 89-115. https://doi.org/10.1007/s11207-0150822-9.

Vršnak B, Warmuth A, Brajša R, Hanslmeier A. 2002. Flare waves observed in Helium I $10830 \AA$ A. A link between $\mathrm{H} \alpha$ Moreton and EIT waves. Astron Astrophys 394: 299-310. https://doi.org/ 10.1051/0004-6361:20021121.

Vršnak B, Warmuth A, Temmer M, Veronig A, Magdalenić J, Hillaris A, Karlický M. 2006. Multi-wavelength study of coronal waves associated with the CME-flare event of 3 November 2003. Astron Astrophys 448(2): 739-752. https://doi.org/10.1051/00046361:20053740.

Wang Y, Shen C, Wang S, Ye P. 2004. Deflection of coronal mass ejection in the interplanetary medium. Sol Phys 222(2): 329-343. https://doi.org/10.1023/B:SOLA.0000043576.21942.aa.

Warmuth A. 2007. Large-scale Waves and Shocks in the Solar Corona. In: The High Energy Solar Corona: Waves, Eruptions, Particles, vol 725, Lecture Notes in Physics, Klein K-L, MacKinnon AL, (Eds.) Springer Verlag, Berlin. pp. 107-138 ISBN 978-3-540-71,569-6.

Warmuth A. 2015. Large-scale Globally Propagating Coronal Waves. Living Rev Sol Phys 12(1): 3. https://doi.org/10.1007/ lrsp-2015-3.
Warmuth A, Vršnak B, Aurass H, Hanslmeier A. 2001. Evolution of Two EIT/H $\alpha$ Moreton Waves. Astrophys J Lett 560: L105-L109. https://doi.org/10.1086/324055.

Warmuth A, Vršnak B, Magdalenić J, Hanslmeier A, Otruba W. 2004. A multiwavelength study of solar flare waves. I. Observations and basic properties. Astron Astrophys 418: 1101-1115. https://doi.org/10.1051/0004-6361:20034332.

Witasse O, Sánchez-Cano B, Mays ML, Kajdič P, Opgenoorth H, et al. 2017. Interplanetary coronal mass ejection observed at STEREO-A, Mars, comet 67P/Churyumov-Gerasimenko, Saturn, and New Horizons en route to Pluto: Comparison of its Forbush decreases at 1.4, 3.1, and 9.9 AU. J Geophys Res (Space Phys) 122(8): 7865-7890. https://doi.org/10.1002/2017JA023884.

Zhang J, Dere KP. 2006. A Statistical study of main and residual accelerations of coronal mass ejections. Astrophys $J$ 649: 1100 1109. https://doi.org/10.1086/506903.

Žic T, Vršnak B, Skender M. 2007. The magnetic flux and selfinductivity of a thick toroidal current. J Plasma Phys J Plasma Phys 73: 741-756. https://doi.org/10.1017/S0022377806006209.

Žic T, Vršnak B, Temmerr M. 2015. Heliospheric propagation of coronal mass ejections: drag-based model fitting. Astrophys $J$ Suppl Ser 218: 32. https://doi.org/10.1088/0067-0049/218/2/32.

Cite this article as: Vršnak B 2021. Analytical and empirical modelling of the origin and heliospheric propagation of coronal mass ejections, and space weather applications. J. Space Weather Space Clim. 11, 34. https://doi.org/10.1051/swsc/2021012. 